\title{
Clinical Characteristics of Colorectal Cancer Patients in terms of Selected Platelet Indices
}

\author{
Angelika Copija $\mathbb{D}^{1,2}$ Ewa Nowakowska-Zajdel, ${ }^{1,2}$ Karolina Janion, ${ }^{1}$ \\ and Katarzyna Walkiewicz $\mathbb{D D}^{2}$ \\ ${ }^{1}$ Department of Nutrition-Related Disease Prevention, Department of Metabolic Disease Prevention, School of Public Health \\ in Bytom, Medical University of Silesia, Katowice, Poland \\ ${ }^{2}$ Department of Clinical Oncology, Regional Specialised Hospital No 4, Bytom, Poland
}

Correspondence should be addressed to Angelika Copija; angelika.copija@gmail.com

Received 6 January 2020; Revised 9 July 2020; Accepted 31 July 2020; Published 9 October 2020

Academic Editor: Hubertus Himmerich

Copyright (C) 2020 Angelika Copija et al. This is an open access article distributed under the Creative Commons Attribution License, which permits unrestricted use, distribution, and reproduction in any medium, provided the original work is properly cited.

\begin{abstract}
Mounting evidence suggests that inflammation, immune response, and coagulation status determine many processes during the carcinogenesis pathway in colorectal cancer (CRC). Inflammation strongly promotes tumor formation, progression, and metastasis. The systemic inflammatory response (SIR) may be reflected by simple indicators evaluated on the basis of peripheral blood morphology parameters. The indices are easily obtained by the peripheral blood test and could be promising biomarkers for CRC. We present the results of the retrospective study evaluating the potential relation between the platelet indices (platelet count (PC), platelet-to-lymphocyte ratio (PLR), neutrophil platelet score (NPS), mean platelet volume (MPV), and MPV/PC ratio) and the clinicopathological features of CRC patients. The study included 247 patients (104 males and 143 females) aged 39-87 years with CRC stages II-IV. The complete blood counts with the automated differential counts were performed prior to the qualification to systemic treatment. High PC, high PLR, and NPS 0 were associated with older age and higher BMI of the patients. No link between the analyzed platelet indices and histological grade of the tumor, primary tumor location, and gender was noted. The patients aged $\geq 65$ years were characterized by the higher MPV/PC ratio than the younger population. We observed a trend to the higher MPV/PC ratio among the patients with excessive body weight defined by BMI compared to BMI within normal limits. A higher frequency of PC > 400, NPS 1 and 2, and a trend to more frequent PLR $\geq 150$ were observed in the subgroup with metastatic disease compared to individuals with CRC stages II and III. The presented results expand the knowledge on potential association between SIR parameters and other clinicopathological factors that should be considered during interpreting the prognostic and predictive value of the inflammation parameters.
\end{abstract}

\section{Introduction}

In 1863, Rudolf Virchow hypothesized that chronic inflammation could sustain cell proliferation contributing to cancerogenesis [1]. Mounting evidence established the role of inflammatory and thrombotic processes in all stages of carcinogenesis in colorectal cancer. The local cancer-related inflammation is reflected in the systemic inflammatory response (SIR).

Besides their physiological role in hemostasis, platelets contribute various pathological processes including inflammation, atherosclerosis, and cancer metastasis via the release of cytokines and chemokines and expression of several adhesion receptors [2]. Tumor cells secrete a plethora of bioactive mediators contributing to platelet activation, e.g., cysteine proteinases, ADP [3] or IL-6 [4]. Platelets may be activated by the production of thrombin stimulated by the tissue factor, upregulated in the oxidative stress in the tumor microenvironment [5]. The colorectal cancer cells and activated platelets may interact using $\alpha \operatorname{IIb} \beta 3$ structures or via $\mathrm{P}$ selectin [5]. Activated platelets secrete several tumor growth and proangiogenic factors involved in the cancerogenesis, including transforming growth factor $\beta$, platelet-derived growth factor, vascular endothelial growth factor, epidermal growth factor, and angiopoietin [6]. Circulating tumor cells avoid the immune surveillance and promote their extravasation and metastasis formation by being coated by blood 
platelets making them unrecognizable for the natural killer cells $[5,7]$.

The potential prognostic role of the combinations of the SIR parameters (such as lymphocytes, neutrophils, and platelets obtained by the peripheral blood test), including the platelet-to-lymphocyte ratio (PLR), neutrophil platelet score (NPS), or mean platelet volume (MPV) to platelet count (PC) ratio, was investigated in various cancers, including CRC [8-10]. However, majority of the studies focused on the primary operable cancer [11-15]; some of them observed patients with advanced diseases [16-18] or patients in all clinical stages [19]. The relation between platelet indices and clinical features is still inconsistent. Moreover, the interpretation is difficult because comorbidities affecting this imbalance may be important in the pathophysiology of SIR.

The aim of the study is to explore the potential relation between the platelet indices (PC, PLR, NPS, MPV, and MPV/PC) and the clinicopathological features of colorectal cancer patients.

\section{Material and Methods}

We conducted the retrospective observational study. Medical records of 247 consecutive patients with histologically confirmed colorectal cancer were evaluated. The study group included patients with disease stages II-IV prior to qualification to systemic treatment (adjuvant or first-line palliative treatment). The established exclusion criteria included the following: other active or past cancers except squamous cell carcinoma of the skin, underwent chemotherapy for CRC during the past 6 months, diagnosed infectious disease (hepatitis B and C, HIV infection or AIDS, and tuberculosis), systemic diseases requiring long-term immunosuppression, cancer cachexia, and no patient consent to participate in the study.

Patients' demographics and clinical and pathological characteristics were collected on the basis of medical history. The peripheral venous blood samples were collected from each patient from a single puncture at a routine blood test necessary for qualification for chemotherapy. Whole blood samples were collected in EDTA-containing tubes, and complete blood counts with automated differential counts were performed.

PLR was calculated by dividing PC by the absolute neutrophil count (NC). Similarly, the MPV/PC ratio was calculated by dividing MPV by the platelet count. NPS was calculated as follows: patients with a neutrophil count $\leq 7.5$ $\times 10^{9} / \mathrm{L}$ and platelets $\leq 400 \times 10^{9} / \mathrm{L}$ scored 0 , patients with neutrophils $>7.5 \times 10^{9} / \mathrm{L}$ or platelets $>400 \times 10^{9} / \mathrm{L}$ scored 1 , and patients with both neutrophils $>7.5 \times 10^{9} / \mathrm{L}$ and platelets $>400 \times 10^{9} / \mathrm{L}$ scored $2[20]$.

2.1. Statistical Analysis. Data were presented as mean \pm standard deviation or median (interquartile range) for continuous variables and frequency or rate for categorical variables. The normal distribution in the analyzed population was tested with the Shapiro-Wilk test. The Mann-Whitney $U$ test and the Kruskal-Wallis $H$ test were used to determine the significance of between-group differences. All statistical analyses were carried out using the Statistica version 13 software package (StatSoft).
TABLE 1: General characteristics of the study group, $n=247$.

\begin{tabular}{lc}
\hline Variable & $n(\%)$ \\
\hline Age (years) & $105(42.5)$ \\
$\leq 65$ & $142(57.6)$ \\
$>65$ & \\
Gender & $104(42.1)$ \\
Male & $143(57.9)$ \\
Female & \\
BMI (kg/m $\left.{ }^{2}\right)$ & $109(44.1)$ \\
$20-25$ & $83(33.6)$ \\
$25-30$ & $55(22.3)$ \\
$\geq 30$ & \\
Grade $(n=200)$ & $16(8)$ \\
I & $138(69)$ \\
II & $46(23)$ \\
III & \\
Stage & $51(20.7)$ \\
II & $65(26.3)$ \\
III & $131(53.0)$ \\
IV & \\
Primary tumor location & $72(29.1)$ \\
Right colon & $121(49.0)$ \\
Left colon & $54(21.9)$ \\
Rectum &
\end{tabular}

\section{Results}

The study group contains 247 patients aged 39-87 years treated in the Department of Clinical Oncology in the period from January 2016 to July 2019. Baseline characteristics of the study group are presented in Table 1.

We observed no association between the analyzed platelet indices and the histological grade of the tumor, primary tumor location (Table 2), and gender.

Patients with metastatic disease had higher WBC and NC compared to individuals with CRC stages II and III after radical resection (Table 3 ). We noted a higher frequency of PC $>400$, NPS 1 and 2, and a trend to more frequent PLR $\geq$ 150 in the subgroup with stage IV disease (Table 4).

High PC (defined according to the literature as PC $>400$ $[9,14,21,22])$ and high PLR (defined as PLR > $150[8,9,13-$ $15,18,22])$ were linked with younger age and lower BMI of the patients (Table 4). Similarly, NPS 0 was related with older age $(p=0.0010)$ and higher BMI $(p=0.0186)$ of patients compared to the subgroup with NPS 1 and 2. No link between MPV and patients' BMI or age was observed. There was a trend to the lower MPV/PC ratio among patients with normal weight compared to overweight and obese patients $(0.028(0.021-0.039)$ vs. $0.030(0.023-0.040)$ vs. 0.033 (0.025-0.046) accordingly, $p=0.052)$. The MPV/PC ratio was higher in patients aged $\geq 65$ years than in the younger population (0.032 (0.024-0.044) vs. 0.028 (0.021-0.037) accordingly, $p=0.009$ ). 
TABLE 2: Characteristics of the study group according to primary tumor location.

\begin{tabular}{|c|c|c|c|c|c|}
\hline \multirow{2}{*}{ Variable } & \multirow{2}{*}{ Total } & \multicolumn{3}{|c|}{ Primary tumor location } & \multirow[b]{2}{*}{$p$} \\
\hline & & RCC & LCC & $\mathrm{RC}$ & \\
\hline Age (years) & $66.02 \pm 9.20$ & $68.67 \pm 7.24$ & $65.13 \pm 9.72$ & $64.48 \pm 9.74$ & 0.0319 \\
\hline BMI $\left(\mathrm{kg} / \mathrm{m}^{2}\right)$ & $26.24 \pm 4.65$ & $26.72 \pm 4.82$ & $25.74 \pm 4.71$ & $26.47 \pm 4.21$ & 0.218 \\
\hline $\mathrm{WBC}\left(\times 10^{9} / \mathrm{L}\right)$ & $6.90(5.65-8.81)$ & $6.56(5.56-8.96)$ & $7.55(5.80-9.12)$ & $6.60(4.40-8.56)$ & 0.094 \\
\hline $\mathrm{NC}\left(\times 10^{9} / \mathrm{L}\right)$ & $4.38(3.27-6.68)$ & $3.94(2.99-5.38)$ & $4.54(3.34-6.03)$ & $4.34(3.52-5.11)$ & 0.203 \\
\hline $\mathrm{PC}\left(\times 10^{9} / \mathrm{L}\right)$ & $276(221-342)$ & $276(216-342)$ & $276(229-341)$ & $277(216-351)$ & 0.678 \\
\hline PLR & $158.5(119.4-237.1)$ & $153.8(11.6-209.1)$ & $155.4(121.8-219.6)$ & $174.1(122.8-308.5)$ & 0.156 \\
\hline MPV (fL) & $8.1(7.1-10.3)$ & $8.2(7.1-10.6)$ & $8.4(7.2-10.5)$ & $7.9(7.0-9.8)$ & 0.351 \\
\hline $\mathrm{MPV} / \mathrm{PC}$ ratio & $0.030(0.023-0.040)$ & $0.030(0.023-0.045)$ & $0.031(0.023-0.041)$ & $0.030(0.021-0.038)$ & 0.629 \\
\hline
\end{tabular}

TABLE 3: Characteristics of the study group according to clinical stage of CRC.

\begin{tabular}{|c|c|c|c|c|c|}
\hline Variable & Total & II $(n=51)$ & $\begin{array}{l}\text { Clinical stage } \\
\text { III }(n=65)\end{array}$ & IV $(n=131)$ & $p$ \\
\hline Age (years) & $66.02 \pm 9.20$ & $65.29 \pm 9.11$ & $65.71 \pm 9.66$ & $66.46 \pm 9.05$ & 0.921 \\
\hline BMI $\left(\mathrm{kg} / \mathrm{m}^{2}\right)$ & $26.24 \pm 4.65$ & $26.04 \pm 4.44$ & $26.78 \pm 4.92$ & $26.05 \pm 4.61$ & 0.695 \\
\hline $\mathrm{WBC}\left(\times 10^{9} / \mathrm{L}\right)$ & $6.89(5.65-8.81)$ & $6.13(5.47-8.01)$ & $6.28(5.03-7.77)$ & $7.94(6.10-10.08)$ & $<0.0001$ \\
\hline $\mathrm{NC}\left(\times 10^{9} / \mathrm{L}\right)$ & $4.38(3.27-5.68)$ & $3.72(2.69-4.70)$ & $3.56(2.89-4.77)$ & $4.80(3.74-6.48)$ & $<0.0001$ \\
\hline $\mathrm{PC}\left(\times 10^{9} / \mathrm{L}\right)$ & $276(221-342)$ & $273(229-342)$ & $264(219-323)$ & $288(220-374)$ & 0.152 \\
\hline PLR & $158.5(119.4-237.1)$ & $138.2(106.4-207.5)$ & $150.8(126.0-237.0)$ & $165.1(125.0-250.9)$ & 0.158 \\
\hline MPV (fL) & $8.1(7.1-10.3)$ & $8.9(7.1-10.2)$ & $7.9(7.2-9.9)$ & $8.3(7.1-10.5)$ & 0.742 \\
\hline MPV/PC ratio & $0.030(0.023-0.040)$ & $0.029(0.025-0.043)$ & $0.032(0.024-0.041)$ & $0.031(0.021-0.039)$ & 0.308 \\
\hline
\end{tabular}

\section{Discussion}

Platelet involvement is observed in almost every step of the metastatic process [5]; hence, platelet indices are potential interesting markers in the metastatic disease. Platelets induce circulating tumor cell epithelial-mesenchymal migration and facilitate tumor cell extravasation and metastasis formation $[6,23,24]$. Experimental studies on animal models indicated the inhibition of pulmonary metastasis by induced thrombocytopenia, which can be reconstituted by platelet infusion [25].

Contrarily, lymphocytes mediate tumor growth control and were inversely related with tumor proliferation and invasiveness [26]. Differentiated CD8+ T cells induce cytotoxic T cell killing and apoptosis. CD4+ T cells play a crucial role in the antitumor immune response. A reduced lymphocyte level facilitates tumor metastatic potential [27]. A decrease in tumor-infiltrating lymphocytes is postulated to be associated with poor survival in CRC [28]. Although no direct research has demonstrated that the peripheral lymphocyte count correlates with the number of tumor-infiltrating lymphocytes, some studies have postulated an association between them [9].

An elevated PLR is a result of an increased number of platelets and/or a decreased number of lymphocytes. Results of a meta-analysis conducted by Chen et al. suggest the association between increased PLR and inferior clinical features of CRC such as poorly differentiated tumor $(\mathrm{OR}=1.51$; 95\% CI, 1.26-1.81; $p<0.001)$, higher tumor stage $(\mathrm{OR}=1.25 ; 95 \% \mathrm{CI}, 1.05-1.49 ; p=0.012)$, lymphovascular invasion $(\mathrm{OR}=1.25 ; 95 \% \mathrm{CI}, 1.09-1.43 ; p=0.001)$, and recurrence of $\mathrm{CRC}(\mathrm{OR}=2.78 ; 95 \% \mathrm{CI}, 1.36-5.68 ; p=0.005$ ) [8], suggesting that PLR could be feasible for tumor staging. Another meta-analysis by Huang et al. confirms the relation between elevated PLR and more advanced clinical stage, $\mathrm{pT}$ category, and degree of differentiation; however the association between lymph node metastasis, lymphatic and venous invasion, and PLR was not observed [9]. Our results do not support these findings; however, in most of the studies included in the meta-analyses, PLR was obtained before the surgery in patients with early-stage CRC while our results are obtained in the population with stage II-IV disease and the majority of the patients had underwent surgery before.

Previous studies showed the association between high PLR and female gender [8] and cancer location in the colon vs. rectum $[8,29]$, which was not confirmed in our results.

The relation between platelet indices and CRC patients' nutritional status has not been sufficiently examined. Obesity is the well-established risk factor for colorectal cancer. Onethird of the patients in the analyzed population were overweight, and one-fifth were obese. We observed lower PC and a trend to lower PLR in patients with excess BMI. These results are puzzling considering the previously described link between increased PC and metabolic syndrome in adults [29]. Increased adipose tissue interferes with platelet function directly by producing adipokines, such as leptin and adiponectin, and inducting chronic systemic low-grade inflammation [30]. Adipose tissue in obese individuals is infiltrated 
TABLE 4: Characteristics of subgroups depending on PC, PLR, and NPS.

\begin{tabular}{|c|c|c|c|c|c|c|c|c|c|c|}
\hline \multirow[b]{2}{*}{ Variable } & \multicolumn{2}{|c|}{$\mathrm{PC}$} & \multirow[b]{2}{*}{$p$} & \multicolumn{2}{|c|}{ PLR } & \multirow[b]{2}{*}{$p$} & \multicolumn{3}{|c|}{ NPS } & \multirow[b]{2}{*}{$p$} \\
\hline & $\begin{array}{l}<400 \\
n(\%)\end{array}$ & $\begin{array}{l}>400 \\
n(\%)\end{array}$ & & $\begin{array}{l}<150 \\
n(\%)\end{array}$ & $\begin{array}{l}\geq 150 \\
n(\%)\end{array}$ & & $\begin{array}{c}0 \\
n(\%)\end{array}$ & $\begin{array}{c}1 \\
n(\%)\end{array}$ & $\begin{array}{c}2 \\
n(\%)\end{array}$ & \\
\hline Age & & & 0.0028 & & & 0.0016 & & & & 0.0004 \\
\hline$<65$ & $81(77.1)$ & $24(22.8)$ & & $35(33.3)$ & $70(66.7)$ & & $73(69.5)$ & $23(21.9)$ & $9(8.6)$ & \\
\hline$>65$ & $129(90.8)$ & $13(9.1)$ & & $76(53.5)$ & $66(46.5)$ & & $127(89.4)$ & $10(7.1)$ & $5(3.5)$ & \\
\hline Gender & & & 0.336 & & & 0.377 & & & & 0.090 \\
\hline Male & $92(87.6)$ & $13(12.4)$ & & $44(41.9)$ & $60(58.1)$ & & $88(83.8)$ & $15(14.3)$ & $2(1.9)$ & \\
\hline Female & $118(83.1)$ & $24(16.9)$ & & $68(47.6)$ & $75(52.4)$ & & $112(78.9)$ & $18(12.7)$ & $12(8.4)$ & \\
\hline BMI & & & 0.0278 & & & 0.0796 & & & & 0.194 \\
\hline$\leq 25$ & $87(79.1)$ & $23(20.9)$ & & $42(38.2)$ & $68(61.8)$ & & $85(77.3)$ & 15 (13.6) & $10(9.1)$ & \\
\hline $25-30$ & $71(86.8)$ & $11(13.4)$ & & $36(47.0)$ & $44(53.0)$ & & $66(80.5)$ & $13(15.9)$ & $3(3.6)$ & \\
\hline$\geq 30$ & $52(94.6)$ & $3(5.4)$ & & $31(56.4)$ & $24(43.6)$ & & $49(89.1)$ & $5(9.1)$ & $1(1.8)$ & \\
\hline Tumor location & & & 0.172 & & & 0.577 & & & & 0.172 \\
\hline RCC & $66(91.7)$ & $6(8.3)$ & & $34(47.2)$ & $38(52.8)$ & & $63(87.5)$ & $8(11.1)$ & $1(1.4)$ & \\
\hline LCC & 99 (81.8) & $22(18.2)$ & & $57(47.1)$ & 64 (52.9) & & $94(77.7)$ & $16(13.2)$ & $11(9.1)$ & \\
\hline $\mathrm{RC}$ & $45(83.3)$ & $9(16.7)$ & & $21(38.9)$ & $33(61.1)$ & & $43(79.6)$ & $9(16.7)$ & $2(3.7)$ & \\
\hline Grade & & & 0.324 & & & 0.596 & & & & 0.760 \\
\hline I & $15(93.8)$ & $1(6.2)$ & & $6(37.5)$ & $10(62.5)$ & & $14(87.5)$ & $2(12.5)$ & $0(0)$ & \\
\hline II & $119(86.2)$ & $19(13.8)$ & & $70(50.7)$ & $68(49.3)$ & & $115(83.3)$ & $16(11.6)$ & $7(5.1)$ & \\
\hline III & $43(93.5)$ & $3(6.5)$ & & $22(47.8)$ & $24(52.2)$ & & $41(89.1)$ & $4(8.7)$ & $1(2.2)$ & \\
\hline Clinical stage & & & 0.0122 & & & 0.063 & & & & 0.0046 \\
\hline II & $47(92.2)$ & $4(7.8)$ & & $29(56.9)$ & $22(43.1)$ & & $47(92.2)$ & $4(7.8)$ & 0 & \\
\hline III & $60(92.3)$ & $5(7.7)$ & & $32(49.2)$ & $33(50.8)$ & & $58(89.3)$ & $6(9.2)$ & $1(1.5)$ & \\
\hline IV & $103(78.6)$ & $28(21.4)$ & & $51(38.60)$ & $81(61.4)$ & & $95(72.5)$ & $23(17.6)$ & $13(9.9)$ & \\
\hline
\end{tabular}

with increased numbers of macrophages producing proinflammatory cytokines [31]. High concentrations of adiposity-related inflammatory factors, i.e., tumor necrosis factor-alpha (TNF- $\alpha$ ), interferon- $\gamma$, and interleukin- (IL-) 1 , 6,8 , and 10 , have been detected both in the adipose tissue and blood [32]. Inflammatory markers such as the highsensitivity C-reactive protein (hsCRP) and white blood cell (WBC) count have been linked with the degree of obesity expressed as the BMI [33], waist circumference (WC) [34], and waist-hip ratio [35]. Some authors suggest the association between the visceral adipose tissue area and the WBC, hsCRP, and NLR levels (but not PLR), whereas no similar observation was confirmed for subcutaneous adipose tissue areas [36]. Recently published findings indicate significantly higher PLR in the colorectal cancer patients with the metabolic syndrome; however, the results were obtained in the group of patients before surgical treatment [29]. On the other hand, results of the population-based survey conducted in almost 40,000 US citizens aged $\geq 60$ years showed that elevated PLR values were significantly associated with the sarcopenia status and negatively associated with the skeletal muscle index [37]. Considering the above results and the fact that the majority of patients with CRC are aged $\geq 60$ years and are often affected by excessive body weight, the interpretation of the described links and the potential diagnostic significance of platelet indices require further research. It remains unclear if the inflammatory processes associated with exceeding from normal weight to overweight in cancer-free individuals differ in terms of pathophysiology from the inflammatory processes associated with colorectal cancer development.

MPV reflects platelet size and is a surrogate of platelet activation $[12,38]$. Decreased MPV may be the effect of increased large platelets in terms of inflammation, possibly because larger platelets are more responsive to stimulation and plenty of larger platelets are selectively degraded in the environment of the neoplasm [10]. Additionally, the inverse relationship between MPV and PC suggests that these two variables should be interpreted as a ratio rather than being used alone [10]. Recent studies showed the higher MPV levels in colorectal cancer patients compared to the control group [12,19] and the reduction of MPV after surgical tumor resection [12]. Based on these observations, MPV could be tested as a potential marker of surveillance in postoperative CRC patients. However, the data on the possible prognostic role of MPV is ambiguous. Some evidence suggests the association between increased MPV and poor overall survival in patients undergoing surgical treatment [39]. On the other hand, decreased pretreatment MPV was showed to predict worse prognosis in the population with advanced CRC qualified to first-line palliative chemotherapy [16]. In the present study, we observed no differences in MPV in CRC patients through TNM stages I-IV which is in agreement with previously published data [19]. $\mathrm{Wu}$ et al. described the significant difference in MPV/PC and TNM stage of CRC (I/II vs. III/IV) and in terms of lymph 
node invasion [10], which was not confirmed in our results. We noted no link between the MPV or MPV/PC ratio and the other inferior clinical features of CRC.

We analyzed NPS postulated by Watt et al. as a promising marker predicting survival in patients undergoing potentially curative surgery for CRC, independent of the TNM stage [20]. In the analyzed population, NPS 1 and 2 were more frequent in patients with metastatic disease. However, due to restrictive criteria in the calculation of NPS, NPS2 was found in a small percentage of cases; therefore, the results of the subgroup analysis within a small study group do not achieve adequate statistical power. Although single reports regarding the use of this parameter in the diagnosis of other gastrointestinal neoplasms have been published (in patients with operable gastric cancer [40] and metastatic pancreatic cancer [41]), no prognostic value of NPS was proven.

\section{Conclusions}

Patients with metastatic disease were more frequently characterized by high PC, high PLR, and NPS 1 and 2 compared to stage II-III disease. The relation between PC, PLR, and NPS and patients' age and BMI was observed. We noted no significant link between analyzed clinicopathological parameters in terms of MPV and MPV/PC.

The interpretation of the present study has a number of possible limitations. First, this was a single-center retrospective study and additional larger validation studies are needed to confirm our results. Second, the data on overall survival is not available yet. Further prospective studies are warranted to assess the exact role of platelet indices in colorectal cancer. Comorbidities were not analyzed because in the authors' opinion the interpretation would be difficult. Not only the presence of comorbidities but also their duration, treatment, and patient lifestyle should be considered. The authors focused on the analysis of SIR with respect to age, sex, BMI, stage, and location of the primary tumor, and most patients in the study group were over 50 years old.

The platelet indices including PLR, NPS, MPV, and MPV/PC may be a promising diagnostic biomarker for CRC. They are inexpensive and widely available. However, many possible medical conditions, including obesity or inflammatory diseases, can potentially affect platelets. Hence, it is particularly important to better understand potential association between SIR parameters and other clinicopathological factors, which should be taken into account when interpreting the results of studies on the prognostic and predictive value of inflammation parameters.

\section{Data Availability}

Requests for additional data or for support with reusing the data should be emailed to the authors, who can be contacted at angelika.copija@gmail.com.

\section{Conflicts of Interest}

The authors declare no conflicts of interest.

\section{Acknowledgments}

Ewa Nowakowska-Zajdel acknowledges the scientific grant from Medical University of Silesia (KNW-1-087/K/9/K).

\section{References}

[1] F. Balkwill and A. Mantovani, "Inflammation and cancer: back to Virchow?," The Lancet, vol. 357, no. 9255, pp. 539-545, 2001.

[2] L. Borsig, "The role of platelet activation in tumor metastasis," Expert Review of Anticancer Therapy, vol. 8, no. 8, pp. 12471255, 2008.

[3] G. Grignani, A. Falanga, and L. Pacchiarini, "Human breast and colon carcinomas express cysteine proteinase activities with pro-aggregating and pro-coagulant properties," International Journal of Cancer, vol. 42, no. 4, pp. 554-557, 1988.

[4] M. F. Neurath and S. Finotto, "IL-6 signaling in autoimmunity, chronic inflammation and inflammation-associated cancer," Cytokine \& Growth Factor Reviews, vol. 22, no. 2, pp. 83-89, 2011.

[5] K. Konstantopoulos and S. N. Thomas, "Cancer cells in transit: the vascular interactions of tumor cells," Annual Review of Biomedical Engineering, vol. 11, no. 1, pp. 177-202, 2009.

[6] M. Labelle, S. Begum, and R. O. Hynes, "Direct signaling between platelets and cancer cells induces an epithelialmesenchymal-like transition and promotes metastasis," Cancer Cell, vol. 20, no. 5, pp. 576-590, 2011.

[7] B. Nieswandt, M. Hafner, B. Echtenacher, and D. N. Männel, "Lysis of tumor cells by natural killer cells in mice is impeded by platelets," Cancer Research, vol. 59, no. 6, pp. 1295-1300, 1999.

[8] N. Chen, W. Li, K. Huang et al., "Increased plateletlymphocyte ratio closely relates to inferior clinical features and worse long-term survival in both resected and metastatic colorectal cancer: an updated systematic review and metaanalysis of 24 studies," Oncotarget, vol. 8, no. 19, pp. 3235632369, 2017.

[9] X. Z. Huang, W. J. Chen, X. Zhang et al., "An elevated plateletto-lymphocyte ratio predicts poor prognosis and clinicopathological characteristics in patients with colorectal cancer: a meta-analysis," Disease Markers, vol. 2017, Article ID 1053125, 10 pages, 2017.

[10] Y.-Y. Wu, X. Zhang, Y. Y. Qin, J. Q. Qin, and F. Q. Lin, “Mean platelet volume/platelet count ratio in colorectal cancer: a retrospective clinical study," BMC Cancer, vol. 19, no. 1, pp. 314$317,2019$.

[11] R. D. Dolan, J. Lim, S. T. McSorley, P. G. Horgan, and D. C. McMillan, "The role of the systemic inflammatory response in predicting outcomes in patients with operable cancer: systematic review and meta-analysis," Scientific Reports, vol. 7, no. 1, article 16717, 2017.

[12] J.-Y. Li, Y. Li, Z. Jiang, R. T. Wang, and X. S. Wang, "Elevated mean platelet volume is associated with presence of colon cancer," Asian Pacific Journal of Cancer Prevention, vol. 15, no. 23, pp. 10501-10504, 2015.

[13] Z. Q. Sun, X. N. Han, H. J. Wang et al., "Prognostic significance of preoperative fibrinogen in patients with colon cancer," World Journal of Gastroenterology, vol. 20, no. 26, pp. 8583-8591, 2014.

[14] C. P. Neal, V. Cairns, M. J. Jones et al., "Prognostic performance of inflammation-based prognostic indices in patients 
with resectable colorectal liver metastases," Medical Oncology, vol. 32, no. 5, 2015.

[15] K. Neofytou, E. C. Smyth, A. Giakoustidis, A. Z. Khan, D. Cunningham, and S. Mudan, "Elevated platelet to lymphocyte ratio predicts poor prognosis after hepatectomy for liveronly colorectal metastases, and it is superior to neutrophil to lymphocyte ratio as an adverse prognostic factor," Medical Oncology, vol. 31, no. 10, pp. 239-239, 2014.

[16] J. Chang, G. Lin, M. Ye et al., "Decreased mean platelet volume predicts poor prognosis in metastatic colorectal cancer patients treated with first-line chemotherapy: results from mCRC biomarker study," BMC Cancer, vol. 19, no. 1, pp. 15-17, 2019.

[17] G. Guo, X. Chen, W. He et al., "Establishment of inflammation biomarkers-based nomograms to predict prognosis of advanced colorectal cancer patients based on real world data," PLoS One, vol. 13, no. 12, article e0208547, 2018.

[18] W. He, C. Yin, G. Guo et al., "Initial neutrophil lymphocyte ratio is superior to platelet lymphocyte ratio as an adverse prognostic and predictive factor in metastatic colorectal cancer," Medical Oncology, vol. 30, no. 1, p. 439, 2013.

[19] M. Stojkovic Lalosevic, A. Pavlovic Markovic, S. Stankovic et al., "Combined diagnostic efficacy of neutrophil-tolymphocyte ratio (NLR), platelet-to-lymphocyte ratio (PLR), and mean platelet volume (MPV) as biomarkers of systemic inflammation in the diagnosis of colorectal cancer," Disease Markers, vol. 2019, Article ID 6036979, 7 pages, 2019.

[20] D. G. Watt, M. J. Proctor, J. H. Park, P. G. Horgan, and D. C. McMillan, "The neutrophil-platelet score (NPS) predicts survival in primary operable colorectal cancer and a variety of common cancers," PLoS One, vol. 10, no. 11, article e0142159, 2015.

[21] V. Josa, M. Krzystanek, A. C. Eklund et al., "Relationship of postoperative thrombocytosis and survival of patients with colorectal cancer," International Journal of Surgery, vol. 18, pp. 1-6, 2015.

[22] J. Zhang, H.-Y. Zhang, J. Li, X.-Y. Shao, and C.-X. Zhang, "The elevated NLR, PLR and PLT may predict the prognosis of patients with colorectal cancer: a systematic review and metaanalysis," Oncotarget, vol. 8, no. 40, pp. 68837-68846, 2017.

[23] A. Passardi, E. Scarpi, L. Cavanna et al., "Inflammatory indexes as predictors of prognosis and bevacizumab efficacy in patients with metastatic colorectal cancer," Oncotarget, vol. 7, no. 22, pp. 33210-33219, 2016.

[24] D. Schumacher, B. Strilic, K. Sivaraj, N. Wettschureck, and S. Offermanns, "Platelet-derived nucleotides promote tumorcell transendothelial migration and metastasis via $\mathrm{P}_{2} \mathrm{Y}_{2}$ receptor," Cancer Cell, vol. 24, no. 1, pp. 130-137, 2013.

[25] S. Karpatkin, E. Pearlstein, C. Ambrogio, and B. S. Coller, "Role of adhesive proteins in platelet tumor interaction in vitro and metastasis formation in vivo," The Journal of Clinical Investigation, vol. 81, no. 4, pp. 1012-1019, 1988.

[26] L. Liang, J. Zhu, H. Jia et al., "Predictive value of pretreatment lymphocyte count in stage II colorectal cancer and in high-risk patients treated with adjuvant chemotherapy," Oncotarget, vol. 7, no. 1, pp. 1014-1028, 2016.

[27] G. P. Dunn, L. J. Old, and R. D. Schreiber, "The immunobiology of cancer immunosurveillance and immunoediting," Immunity, vol. 21, no. 2, pp. 137-148, 2004.

[28] H. A. K. JC, G. R. Guerra, T. Pham, C. Mitchell, A. C. Lynch, and R. R. G. Warrier, "Prognostic impact of tumor- infiltrating lymphocytes in primary and metastatic colorectal cancer," Diseases of the Colon and Rectum, vol. 62, no. 4, pp. 498-508, 2019.

[29] J. You, H. Zhang, Y. Shen et al., "Impact of platelet to lymphocyte ratio and metabolic syndrome on the prognosis of colorectal cancer patients," Oncotargets and Therapy, vol. Volume 10, pp. 2199-2208, 2017.

[30] D. R. Faber, P. G. De Groot, and F. L. J. Visseren, "Role of adipose tissue in haemostasis, coagulation and fibrinolysis," Obesity Reviews, vol. 10, no. 5, pp. 554-563, 2009.

[31] S. P. Weisberg, D. McCann, M. Desai, M. Rosenbaum, R. L. Leibel, and A. W. Ferrante Jr., "Obesity is associated with macrophage accumulation in adipose tissue," The Journal of Clinical Investigation, vol. 112, no. 12, pp. 1796-1808, 2003.

[32] Z. Liu, R. S. Brooks, E. D. Ciappio et al., "Diet-induced obesity elevates colonic TNF- $\alpha$ in mice and is accompanied by an activation of Wnt signaling: a mechanism for obesity-associated colorectal cancer," The Journal of Nutritional Biochemistry, vol. 23, no. 10, pp. 1207-1213, 2012.

[33] C. M. Kitahara, H. Trabert, H. A. Katki et al., "Body mass index, physical activity, and serum markers of inflammation, immunity, and insulin resistance," Cancer Epidemiology, Biomarkers \& Prevention, vol. 23, no. 12, pp. 2840-2849, 2014.

[34] D. Ackermann, J. Jones, J. Barona et al., "Waist circumference is positively correlated with markers of inflammation and negatively with adiponectin in women with metabolic syndrome," Nutrition Research, vol. 31, no. 3, pp. 197-204, 2011.

[35] B. Faam, M. Zarkesh, M. S. Daneshpour, F. Azizi, and M. Hedayati, "The association between inflammatory markers and obesity-related factors in Tehranian adults: Tehran lipid and glucose study," Iranian Journal of Basic Medical Sciences, vol. 17, no. 8, pp. 577-582, 2014.

[36] J. Y. Yu, W. J. Choi, H. S. Lee, and J. W. Lee, "Relationship between inflammatory markers and visceral obesity in obese and overweight Korean adults: an observational study," Medicine, vol. 98, no. 9, article e14740, 2019.

[37] F. Y. Liaw, C. F. Huang, W. L. Chen et al., "Higher platelet-tolymphocyte ratio increased the risk of sarcopenia in the community-dwelling older adults," Scientific Reports, vol. 7, no. 1, article 16609, 2017.

[38] Y. Park, N. Schoene, and W. Harris, "Mean platelet volume as an indicator of platelet activation: methodological issues," Platelets, vol. 13, no. 5-6, pp. 301-306, 2009.

[39] N. Li, Z. Yu, X. Zhang et al., "Elevated mean platelet volume predicts poor prognosis in colorectal cancer," Scientific Reports, vol. 7, no. 1, article 10261, 2017.

[40] Y. Okugawa, Y. Toiyama, A. Yamamoto et al., "Modified neutrophil-platelet score as a promising marker for stratified surgical and oncological outcomes of patients with gastric cancer," Surgery Today, vol. 50, no. 3, pp. 223-231, 2020.

[41] M. Dogan, E. Algin, Z. T. Guven et al., "Neutrophil-lymphocyte ratio, platelet-lymphocyte ratio, neutrophil-platelet score and prognostic nutritional index: do they have prognostic significance in metastatic pancreas cancer?," Current Medical Research and Opinion, vol. 34, no. 5, pp. 857-863, 2018. 